\title{
Mouse interleukin-12/FasTI: A novel bi-functional fusion protein for cancer immuno/gene therapy
}

\author{
$\mathrm{XI} \mathrm{YANG}^{1}$, ASHLEE H. TIETJE $^{2}$, XIANZHONG YU ${ }^{1}$ and YANZHANG WEI ${ }^{1}$ \\ ${ }^{1}$ Department of Biological Sciences, Clemson University, Clemson; \\ ${ }^{2}$ Division of Science, Southern Wesleyan University, Central, SC 29634, USA
}

Received January 6, 2016; Accepted February 17, 2016

DOI: $10.3892 /$ ijo.2016.3475

\begin{abstract}
Whereas cancer immunotherapy with cytokines in recent research was demonstrated effective in activating immune response against tumor cells, one major obstacle with the use of these cytokines is their severe side effects when delivered systemically at high doses. Another challenge is that advanced tumor cells often evade immunosurveillance of the immune system as well as of the Fas-mediated apoptosis by various mechanisms. We report the design and preliminary evaluation of the antitumor activity of a novel fusion proteinmIL-12/FasTI, consisting of mouse interleukin-12 and the transmembrane and intracellular domains of mouse Fas. The fusion construct (pmIL-12/FasTI) was transfected into mouse lung carcinoma cell line TC-1. Stable cell clones expressing the fusion protein were established as assayed by RT-PCR and immunohistochemistry. ELISA and cell proliferation analyses demonstrated that NK cells were effectively activated by the fusion protein with increased IFN- $\gamma$ production and cytotoxicity. Enhanced caspase-3 activity of the clones when co-cultured with NK cells indicated that apoptosis was induced through Fas/FasL signaling pathway. The preliminary results suggest a synergized anticancer activity of the fusion protein. It may represent a promising therapeutic agent for cancer treatment.
\end{abstract}

\section{Introduction}

Natural killer (NK) cells are an important component of the innate immune system that provides rapid responses to malignantly transformed or infected cells through immunosurveillance. NK cells also help to shape the adaptive immunity by interacting with dendritic cells and producing the key cytokine IFN- $\gamma$. The NK cell lineage has been considered for cancer eradication owing to its ability to kill a wide variety of tumor cells spontaneously while sparing normal cells $(1,2)$. Advanced tumor cells, on the contrary, are able to inhibit NK cells and other antitumor immune cells in a tumor microenvi-

Correspondence to: Dr Y. Wei, Department of Biological Sciences, Clemson University, 132 Long Hall, Clemson, SC 29634, USA E-mail: ywei@clemson.edu

Key words: interleukin-12, Fas, natural killer cell, apoptosis ronment, where the cytokine profile favors tumor growth. One of the challenges ahead of cancer immunologists is how to reshape the tumor microenvironment to favor killer cells, such as NK cells, cytotoxic T lymphocytes (CTLs), and $\gamma \delta \mathrm{T}$ cells.

IL-12, also known as NK cell stimulatory factor 2, is an activator and initiator of NK cell activation $(3,4)$. One of the major functions of IL-12 is to enhance NK cell-mediated IFN $-\gamma$ production, which promotes host responses against tumors and regulates tumor development by interacting with its receptor. IL-12 also contributes to activation of $\mathrm{CD} 8^{+} \mathrm{T}$ cells in absence of helper T cells and NK cells. IL-12 has, therefore, been selected as a good candidate for cancer cytokine therapies. In experimental tumor models, recombinant IL-12 treatment has a marked antitumor effect on transplantable tumors, chemically-induced tumors, and in tumors arising spontaneously in genetically modified mice. IFN- $\gamma$ and a cascade of other pro-inflammatory cytokines induced by IL-12 have a direct toxic effect on the tumor cells or may activate potent anti-angiogenic mechanisms (5). However, the IL-12 systemic high toxicity hindered its clinical applications $(6,7)$. Efforts have been made to generate local high dose of IL-12 therapy. Previous studies have successfully extended the glycosylphosphatidylinositol (GPI) anchor technology to murine IL-12. A fusion gene consisting of murine IL-12 cDNA and a GPI-anchoring signal from decay accelerating factor (DAF) showed significant synergistic antitumor effect when co-expressed with GPI-anchored IL-2 $(8,9)$.

Fas (CD95) is a transmembrane cell surface death receptor that belongs to the tumor necrosis factor (TNF) receptor superfamily. Fas contains three cysteine-rich extracellular domains at the $\mathrm{N}$-terminus, which are responsible for ligand binding, the transmembrane domain, and an intracellular death domain (DD) that is essential for transducing apoptotic signals (10). Binding of Fas ligand to Fas receptor results in the recruitment of Fas-associated death domain (FADD) through DD-DD interactions. The death-effector domain of FADD then recruits pro-caspase-8/10 to the receptor, resulting in the formation of death-inducing signaling complex (DISC). DISC activates effector caspases such as caspase-3 that in turn cleaves a restricted set of targets proteins and is responsible for apoptosis in the cells (11).

In this study, we intended to combine the NK cell-mediated cytotoxicity and Fas-mediated apoptosis into one fusion protein by constructing a fusion gene containing cDNAs encoding 
mouse IL-12 followed by the transmembrane and intracellular domains of Fas. We hypothesized that the tumor cells expressing the fusion protein will not only send an apoptotic signal to tumor cells but also activate the NK cells through IL-12 receptor. Therefore, not only the engaged tumor cells will be killed via Fas-induced mechanisms, the neighboring tumor cells that do not express the protein will also be killed by activated NK cells $(12,13)$.

\section{Materials and methods}

Cells. The mouse lung carcinoma TC-1 cells (ATCC no. CRL-2785) were cultured in RPMI-1640 medium containing $10 \%$ fetal bovine serum and $100 \mu \mathrm{g} / \mathrm{ml}$ gentamicin at $37^{\circ} \mathrm{C}$ with $5 \% \mathrm{CO}_{2}$. The human natural killer cell line NK92 cells (ATCC no. CRL-2407) were cultured in aMEM containing $12.5 \%$ fetal bovine serum, $12.5 \%$ horse serum, $2 \mathrm{mM}$ L-glutamine, $1.5 \mathrm{~g} / 1$ sodium bicarbonate, $0.02 \mathrm{mM}$ folic acid, $0.1 \mathrm{mM} 2$-mercaptoethanol, $100 \mu \mathrm{g} / \mathrm{ml}$ gentamicin and $4 \mathrm{ng} / \mathrm{ml}$ interleukin-2 at $37^{\circ} \mathrm{C}$ with $5 \% \mathrm{CO}_{2}$.

Construction of pcDNA3.1 (+) mIL-12/FasTI/Zeo vector. Mouse IL-12 cDNA was cloned from pORF-mIL-12 (InvivoGen, CA, USA), and the transmembrane/intracellular domains of Fas was amplified from a previous pcDNA3.1 (+) MULT1E/FasTI/ Zeo expression vector (12) using 5' primer (CGGGATCCCCC AGAAATCGCCTATGGTTGTTGACC) and 3' primer (CGG AATTCTCACTCCAGACATTGTCCTTCATTTTC). To allow a continuous open reading frame the mIL-12 stop codon was removed from the 3 '-end of the sequence during PCR amplification and replaced with a $(\mathrm{GGGS})_{3}$ linker sequence (GGTGGTGGTTCTGGTGGTGGTTCTGGTGGTGGTTCT) to separate the two functional domains of fusion protein to maintain original folding and binding ability of the proteins. HindIII, BamHI, EcoRI restriction cutting sites were designed for 5'-end of mIL-12, 3'/5'-ends of mIL-12 and FasTI and 3'-end of FasTI, respectively. The primers are: 5'IL-12, CCAAGC TTCCATGGGTCAATCACGCTACCTCC; 3'IL-12, CGCG GATCCAGAACCACCACCAGAACCACCACCAGAACCA CCACCGGATCGGACCTGCAGGGAACACATGC; 5'FasTI, CGGGATCCCCCAGAAATCGCCTATGGTTGTTGACC; 3'FasTI, CGGAATTCTCACTCCAGACATTGTCCTTCA TTTC. The sequence of construct was confirmed using DNA Sanger sequencing.

Construction of pcDNA3.1(+)/mIL-12/Zeo vector (containing PDGRF transmembrane domain). In order to create a cell membrane anchored form of mouse IL-12, the IL-12 sequence was amplified from pORF-mIL-12 using 5' primer (CCCCCCG GGAGGGTCATTCCAGTCTCT) with XmaI restriction cutting site and a $3^{\prime}$ primer (TCCCCGCGGGGATCGGAC CCTGCAGGGAAC) with a SacII restriction cutting site by PCR. The fragments were excised and gel purified using a gel purification kit (Qiagen, Valencia, CA, USA). Double enzyme digestion was performed on the purified fragments of mIL-12 and pDisplay vector (Invitrogen Life Technologies) using $X m a I$ and SacII allowing for the insertion of mIL-12 into the vector. The enzyme digested mIL-12 fragment and pDisplay vector fragment were ligated to create the plasmid pDisplay $(+)$ mIL-12. In order to make the expression vector zeocin resis- tant, restriction enzymes HindIII and XhoI were used to cut plasmid pDisplay (+) mIL-12 and fragment containing mIL-12 sequence together with the cell membrane anchoring sequence was retrieved and inserted into plasmid pcDNA3.1/Zeo(+) to have plasmid pcDNA3.1(+)/mIL12/Zeo. The sequence of construct was confirmed using DNA Sanger sequencing.

Transfection of cells. TC-1 cells were transfected with pcDNA3.1 (+) mIL-12/FasTI/Zeo or pcDNA3.1 (+) mIL-12/ Zeo using Lipofectamine 2000 (Invitrogen) as directed by the manufacturer. To obtain stable clones expressing fusion protein or control protein, the transfected TC-1 cells were cultured in selecting medium containing $200 \mu \mathrm{g} / \mathrm{ml}$ zeocin. Drug-resistance clones were collected and sub-cultured in the presence of the selective drug.

$R T-P C R$. Total RNA was extracted from each clone using an RNeasy Plus from Qiagen according to the manufacturer's instructions. cDNAs were produced from $2 \mu \mathrm{g}$ of the total RNA using the QuantiTect RT kit from Qiagen according to the manufacturer's instructions. Five microliters of the cDNA product were added to a PCR reaction containing primers amplifying a 1059-bp portion of mIL-12/FasTI fusion gene sequence containing 522 bp of the mIL-12, the linker (GGTG GTGGTTCTGGTGGTGGTTCTGGTGGTGGTTCT, and the entire 495 bp of FasTI. A fusion high-fidelity DNA polymerase kit was used with a 5' primer GCAGTGACATGTG GAATGGC and a 3' primer CGGAATTCTCACTCCAGACA TTGTCCTTCATTTTC. Similar reaction was also carried out to amplify portion of mIL-12 sequence using 5' primer GGA AGCACGGCAGCAGAATA and 3' primer AACTTGAGG GAGAAGTAGGAATGG to amplify a 180-bp fragment of mIL-12. $\beta$-actin housekeeping gene was also amplified as controls.

Immunohistochemistry (IHC). Glass slides with cells of clones IL-12/8, IL12-Fas/9, IL12-Fas/10 and TC-1 were prepared using cytospin technology. A hundred thousand cells in $1 \mathrm{ml}$ PBS were transferred into assembled cytospin cassettes and centrifuged in the CytoTek centrifuge at 2,000 rpm for $10 \mathrm{~min}$ at room temperature. After centrifugation the cells on the slides were fixed with $4 \%$ paraformaldehyde (PFA) solution for $10 \mathrm{~min}$. The cells were then stained at room temperature for $1 \mathrm{~h}$ with isotype rat $\mathrm{IgG}_{2 \mathrm{a}}$ antibody (R\&D Systems, clone no. 54447) or anti-mouse IL-12/p35 antibody (R\&D Systems, clone no. 45806) after a 1:500 dilution with sterile PBS. After washing, secondary antibody conjugated with HSS-HRP was added to the cells and incubated for $30 \mathrm{~min}$ at room temperature. DAB substrate-chromogen mixture was applied to the cells and incubated for $10 \mathrm{~min}$. Slides were washed three times using fresh PBS. The slides were examined under a microscope (Olympus 1x70 fluorescent microscope).

Cell proliferation assay. To determine if the clones grow at the same rate as untransfected TC-1 cells, cell proliferation was checked with the MTS assay every $24 \mathrm{~h}$ for 5 days. On day 1, 4,000 cells of each clone were plated on 12-well-plates in triplicates. At each time-point, 3 wells of each clone were tested with MTS. The test was performed twice to obtain 6 replicates. Clones were compared using a one-way ANOVA with Tukey's post-hoc test. 

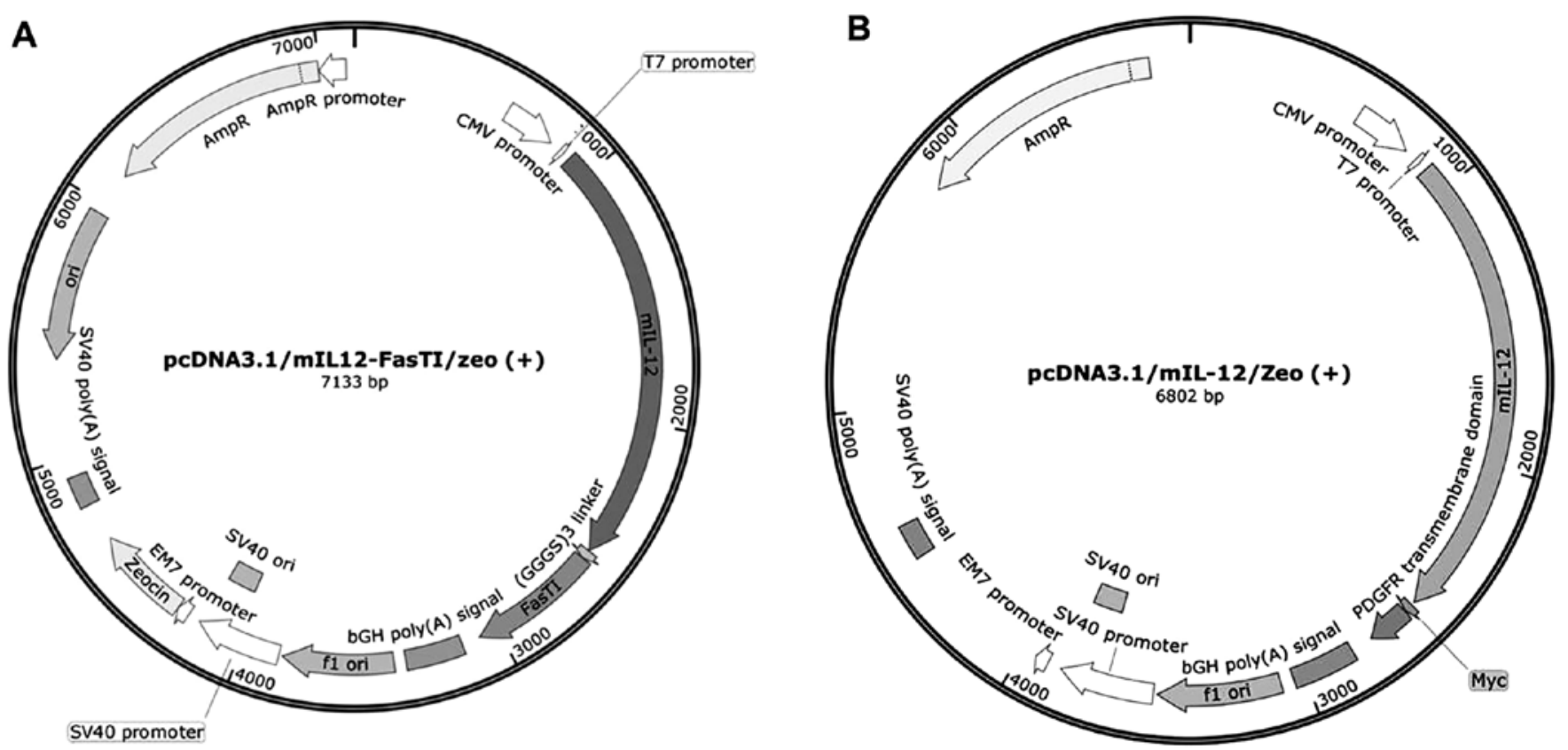

Figure 1. (A) Plasmid pcDNA3.1 (+) mIL-12/FasTI/Zeo. (B) Plasmid pcDNA3.1 (+) mIL-12/Zeo (containing PDGFR transmembrane domain).

NK cell activation. Human NK92 cells and the cells of each clone at a ratio of 10:1 were plated on 96-well plate in $200 \mu \mathrm{l}$ NK media and incubated for $48 \mathrm{~h}$. After incubation $100 \mu \mathrm{l}$ of supernatant was collected from each well and analyzed for the presence of IFN- $\gamma$ using BD OptiEIA human IFN- $\gamma$ ELISA kit II according to the manufacturer's instructions. The rest of the media as well as NK92 cells were removed by PBS washing. The proliferation of the remaining tumor cells was determined using Promega's CellTiter 96 AQueous non-radioactive cell proliferation assay according to the manufacturer's instructions. The data were analyzed using a one-way ANOVA with Tukey's post-hoc test.

Apoptosis assay. One million cells of each clone were first plated on a 6-well-plate and incubated for $24 \mathrm{~h}$. At this point one million NK92 cells were added to the tumor cells and incubated for $2 \mathrm{~h}$. After removing NK cells, apoptosis of the tumor cells was determined by a caspase-3 assay kit (Abcam) according to the manufacturer's instructions.

\section{Results}

\section{Fusion gene construction}

Plasmid pcDNA3.1 (+) mIL-12/FasTI/Zeo. Mouse IL-12 cDNA was amplified from pORF-mIL-12 by PCR and cloned into mammalian expressing vector pcDNA3.1(+)/Zeo with enzyme sites of HindIII and BamHI. The transmembrane/intracellular domains of mouse Fas was amplified from a previous plasmid made in our lab: pcDNA3.1 (+) MULT1E/FasTI/Zeo and cloned into the above vector with enzyme sites of BamHI and $E c o$ RI. To allow an overall open reading frame and functional protein folding, the mIL-12 stop codon was removed and replaced with a (GGGS) $)_{3}$ linker sequence. The fusion gene sequence of the resulting plasmid pcDNA3.1 (+) mIL-12/ FasTI/Zeo (Fig. 1A) was confirmed by DNA sequencing.

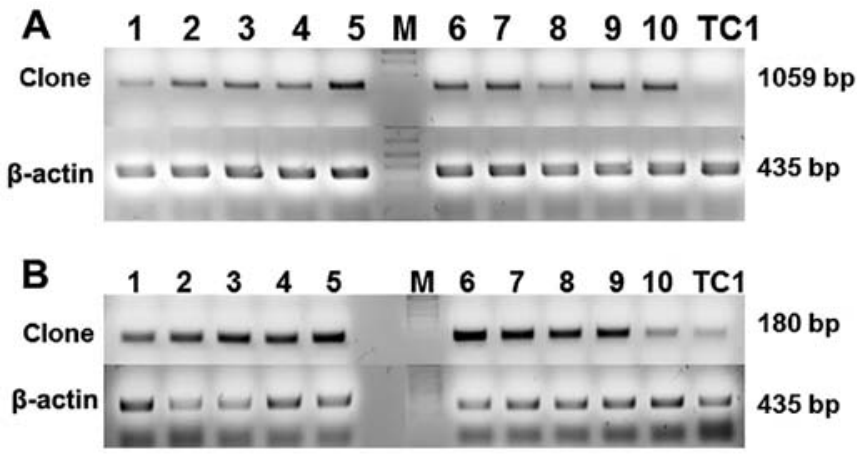

Figure 2. RT-PCR confirmation of gene expression in selected TC-1 clones. A two-step RT-PCR was performed with total RNAs isolated from the clones. (A) Detection of fusion gene MIL-12/FasTI expression. (B) Detection of IL-12 expression. House-keeping gene $\beta$-actin expression was also included as controls.

Plasmid pcDNA3.1 (+) mIL-12/Zeo. The IL-12 cDNA sequence without stop codon was amplified from pORF-mIL-12 and cloned into vector pDisplay with enzyme sites of XmaI and SacII. In order to make the expression vector zeocin resistant, restriction enzymes HindIII and XhoI were used to cut plasmid pDisplay (+) mIL-12 and the fragment containing mIL-12 sequence together with the cell membrane anchoring sequence was retrieved and inserted into plasmid pcDNA3.1/ Zeo. The sequence of the resulting plasmid pcDNA3.1 (+)/ IL-12 (Fig. 1B) was confirmed by DNA sequencing.

Stable clone establishment. TC-1 lung carcinoma cells were transfected with pcDNA3.1 (+) mIL-12/FasTI/Zeo or pcDNA3.1 (+) mIL-12/ Zeo. Ten zeocin resistant clones were selected for each transfection, and the transgene expression was analyzed by RT-PCR. All ten clones are positive for fusion protein IL12/FasTI (Fig. 2A) or cytokine IL12 (Fig. 2B). The 


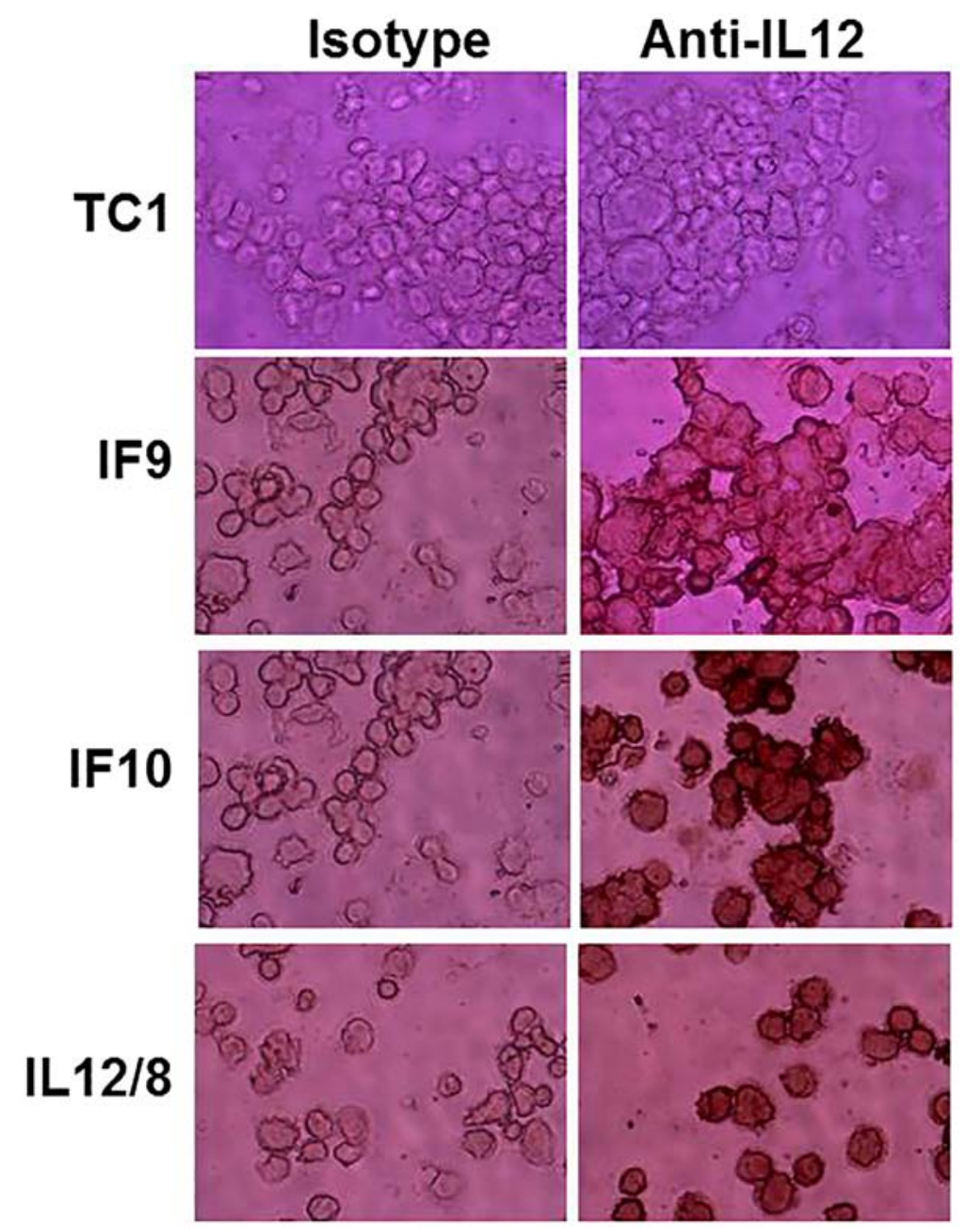

Figure 3. Immunohistochemistry analysis of fusion gene expression. Cell clones and TC-1 cells were cytospun onto class slides. After fixation, the cells were stained with anti-mouse IL-12 antibody or isotype control antibody followed by biotinylated secondary antibody conjugated with HRP. The cells were then treated with substrate DAB and observed under a microscope.

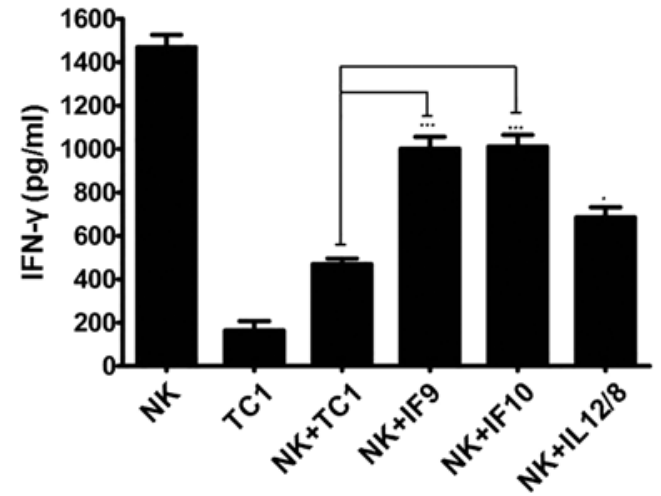

Figure 4. IFN- $\gamma$ production by human NK92 cells. Cell clones and TC-1 in triplicates were co-cultured with human NK92 cells and the supernatants were collected. IFN- $\gamma$ activity in the supernatants was detected using human IFN- $\gamma$ ELISA kit. The statistical analyses were conducted between the controls (NK+TC1) and IL-12 expressing control and IL12-FasTI expressing clones using one-way analysis of variance (ANOVA) with Tukey's post-hoc test $\left(\mathrm{p}<0.05 ;{ }^{* * * *} \mathrm{p}<0.001\right)$.

clones were then examined for protein expression by IL-12 immunohistochemistry (IHC). IHC results demonstrated that fusion clones of IF-9, IF-10 and IL-12 clone IL12/8 expressed relatively high levels of the fusion protein or IL-12 on cell surface compared to control TC-1 cells (Fig. 3).

NK cell activation. It is critical to know whether fusion protein IL12/FasTI can effectively activate NK cells. Cells from TC-1 or clones of IF-9, IF-10 and IL12/8 were co-cultured with human NK92 cells. After the co-culture, the IFN- $\gamma$ production in the supernatant was detected by human IFN- $\gamma$ ELISA analysis and the cytotoxicity of tumor cells was analyzed using the Promega CellTiter 96 AQueous non-radioactive cell proliferation assay. The production of IFN- $\gamma$ was significantly increased by NK cells co-cultured with clone IF-9, IF-10 and IL12/8 compared to those co-cultured with TC-1 parental cells $(p<0.001, p<0.001$ and $p<0.05$, respectively) (Fig 4). Importantly, fusion protein IL-12/FasTI significantly enhanced the cytotoxicity of NK cells (Fig. 5). NK cells co-cultured with clone IF9 and IF10 killed significantly more tumor cells $(\mathrm{p}<0.001$ and $\mathrm{p}<0.001)$ when compared with NK cells co-cultured with control TC-1 cells. It is of interest that although NK cells co-cultured with clone IL12/8, which express only IL-12 on cell surface, killed significant tumor cells $(p<0.01)$, the cytotoxicity is relatively lower than NK cells co-cultured with fusion protein expressing cells. 


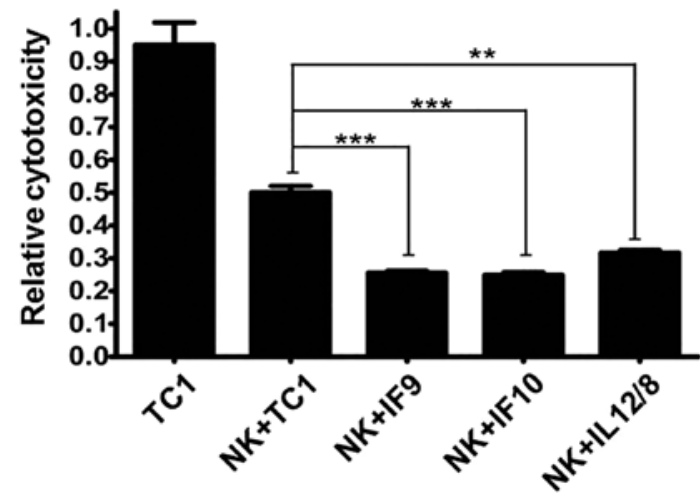

Figure 5. Cytotoxicity of NK cells. Cell clones and TC-1 in triplicates were co-cultured with human NK92 cells. After co-culture, NK cells were removed and remaining tumor cell proliferation was measured with CellTiter 96 AQueous non-radioactive cell proliferation assay kit. The statistical analyses were conducted between the controls $(\mathrm{NK}+\mathrm{TC} 1)$ and IL-12 expressing control and IL12-FasTI expressing clones using one-way analysis of variance (ANOVA) with the Tukey's post-hoc test ${ }^{* *} \mathrm{p}<0.01$, $\left.{ }^{* * *} \mathrm{p}<0.001\right)$

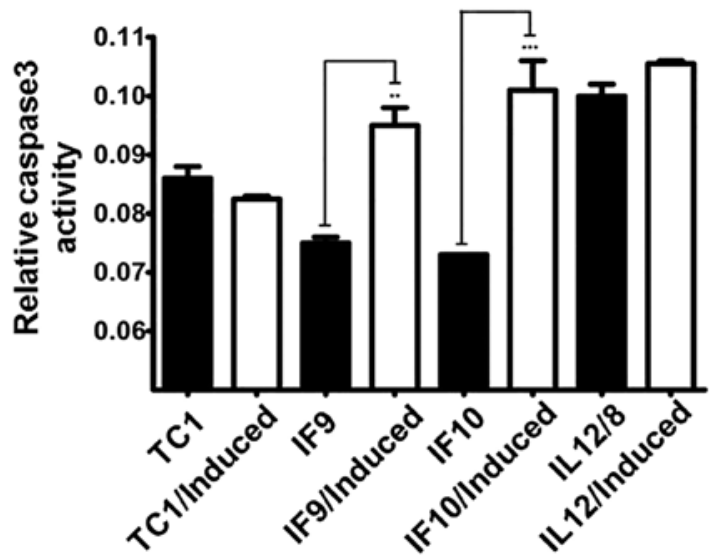

Figure 6. Induction of apoptosis in tumor cells. A total of $1 \times 10^{6}$ cells of TC-1 and clones IF9, IFIO and IL-12/8 in triplicates were co-cultured with NK92 cells for $2 \mathrm{~h}$. After the removal of NK cells, the caspase-3 activity in the remaining tumor cells was detected using a caspase- 3 assay kit. The statistical analyses were conducted between the corresponding un-induced and induced groups using one-way analysis of variance (ANOVA) with the Tukey's post-hoc test $\left({ }^{* *} \mathrm{p}<0.01,{ }^{* * *} \mathrm{p}<0.001\right)$.

Fusion protein IL-12/FasTI induces apoptosis of tumor cells. To confirm the hypothesis that when bound to IL-12 receptor, fusion protein IL-12/FasTI can also send death signals through its FasTI portion into the tumor cells, TC-1 cells and clones IF9, IF10 and IL12/8 were co-cultured with NK cells to induce apoptosis. Caspase-3 assay was performed to analyze the apoptosis in tumor cells. After co-culture, the caspase-3 activity of clone IF9 and IF10 was significantly higher than the un-induced controls ( $\mathrm{p}<0.01$ and $\mathrm{p}<0.001$, respectively). As expected, TC-1 and clone IL12/8 showed no significant difference with or without NK cell co-culture (Fig. 6).

\section{Discussion}

Different strategies using NK cells for antitumor immunotherapy such as in vivo cytokine therapy to expand and activate NK cells against tumor cells have been used $(14,15)$. However, a majority of these studies failed to demonstrate significant clinical benefit. As tumors have developed multiple mechanisms to subvert and suppress immune responses by deregulating cell surface expression of Fas and Fas ligand resulting in reduced sensitivity of tumor cells to Fas-mediated apoptosis. This resistance to Fas-mediated apoptosis protects tumor cells from killing by infiltrating antitumor NK cells and $\mathrm{T}$ cells (16). NK cells require activating receptors as well as activating cytokine, like IL-12, to function. The systemic infusion of IL-12 in high amounts is effective in activating an immune response against tumor cells but it is toxic to most patients. In this study, we introduced mouse IL-12, one of most important and potent NK cell activating cytokines, and the DD of Fas in the transmembrane and intracellular domain into one fusion protein IL-12/FasTI. It is hypothesized that the development of this bi-functional chimeric protein can send an apoptosis signal to the tumor cells and at the same time activate NK cells and other immune cells.

The transcription of fusion protein in zeocin resistant clones was confirmed by RT-PCR (Fig. 2) and the expression of the fusion protein was further characterized by cell surface immunohistochemistry (IHC, Fig. 3). In the RT-PCR experiment, a pair of primers were designed to cover the entire IL-12 fragment and partial upstream fragment of FasTI; thus only clones expressing fusion protein should show a band. In the IHC analysis, when anti-mouse IL12 antibody was used, clones IF-9, IF-10 and IL-12/8 showed positive, whereas TC-1 cells were negative (Fig. 3).

Increased production of IFN- $\gamma$ is a key indicator of $\mathrm{NK}$ cell activation (17). To confirm the idea that fusion protein IL12/FasTI activates immune cells that express IL-12R, human NK92 cells were co-incubated with cells of TC-1 and clones IF-9, IF-10 and IL-12/8 and IFN- $\gamma$ expression was detected by human IFN- $\gamma$ ELISA. The IFN- $\gamma$ expression by NK cells co-cultured with cells of clones IF-9, IF-10 and IL-12/8 increased significantly compared to those of NK cells co-cultured with cells of TC-1 (Fig. 4). Interestingly, NK cells without co-culture produced the most IFN- $\gamma$. This might be due to the fact that tumor cells express ligands for inhibitory receptors on NK cells (18). Therefore, the logic comparison should be between NK cells co-cultured with TC-1 and clones expressing the fusion genes or IL-12. More importantly, the cytotoxicity assay further demonstrated that fusion protein clones IF-9 and IF-10 are more effective in inducing NK mediated cell killing than IL-12/8 clone expressing membrane anchored IL-12. This may be due to the additive effect of FasTI induced apoptosis (Fig. 5).

Another key feature of the designed fusion protein is to send apoptosis signals into the cells expressing the protein after binding to its ligand. Although we do not have direct evidence supporting that the binding of IL-12 to IL-12R on NK cells can trigger FasTI to form DISC inside tumor cells, a clear apoptosis signal is sent to targeted tumor cells as indicated by the increased caspase- 3 activity after co-culture of the clones with NK cells (Fig. 6). This also agrees with the results of cytotoxicity study (Fig. 5).

In conclusion, a bi-functional chimeric protein containing the extracellular domain of mouse IL-12 and transmembrane and intracellular domains of Fas was created. In vitro studies 
demonstrate that the fusion protein can activate NK cells for antitumor cytotoxicity and induce apoptosis in tumor cells. When combined with an effective tumor specific gene delivery vehicle, the bi-functional fusion gene represents a novel approach for cancer immunotherapy.

\section{References}

1. Smyth MJ, Thia KYT, Cretney E, Kelly JM, Snook MB, Forbes CA and Scalzo AA: Perforin is a major contributor to NK cell control of tumor metastasis. J Immunol 162: 6658-6662, 1999.

2. Smyth MJ, Swann J, Cretney E, Zerafa N, Yokoyama WM and Hayakawa Y: NKG2D function protects the host from tumor initiation. J Exp Med 202: 583-588, 2005.

3. Robertson MJ, Soiffer RJ, Wolf SF, Manley TJ, Donahue C, Young D, Herrmann SH and Ritz J: Response of human natural killer (NK) cells to NK cell stimulatory factor (NKSF): Cytolytic activity and proliferation of NK cells are differentially regulated by NKSF. J Exp Med 175: 779-788, 1992.

4. Rodolfo M and Colombo MP: Interleukin-12 as an adjuvant for cancer immunotherapy. Methods 19: 114-120, 1999.

5. Colombo MP and Trinchieri G: Interleukin-12 in anti-tumor immunity and immunotherapy. Cytokine Growth Factor Rev 13: 155-168, 2002.

6. Atkins MB, Robertson MJ, Gordon M, Lotze MT, DeCoste M DuBois JS, Ritz J, Sandler AB, Edington HD, Garzone PD, et al: Phase I evaluation of intravenous recombinant human interleukin 12 in patients with advanced malignancies. Clin Cancer Res 3: 409-417, 1997.

7. Car BD, Eng VM, Lipman JM and Anderson TD: The toxicology of interleukin-12: A review. Toxicol Pathol 27: 58-63, 1999.

8. Ji J, Li J, Holmes LM, Burgin KE, Yu X, Wagner TE and Wei Y Glycoinositol phospholipid-anchored interleukin 2 but secreted interleukin 2 inhibits melanoma tumor growth in mice. Mol Cancer Ther 1: 1019-1024, 2002.
9. Ji J, Li J, Holmes LM, Burgin KE, Yu X, Wagner TE and Wei Y: Synergistic anti-tumor effect of glycosylphosphatidylinositolanchored IL-2 and IL-12. J Gene Med 6: 777-785, 2004.

10. Peter ME and Krammer PH: The CD95(APO-1/Fas) DISC and beyond. Cell Death Differ 10: 26-35, 2003.

11. Houston A and O'Connell J: The Fas signalling pathway and its role in the pathogenesis of cancer. Curr Opin Pharmacol 4: 321-326, 2004

12. Kotturi HSR, Li J, Branham-O'Connor M, Stickel SL, Yu X, Wagner TE and Wei Y: Tumor cells expressing a fusion protein of MULT1 and Fas are rejected in vivo by apoptosis and NK cell activation. Gene Ther 15: 1302-1310, 2008.

13. Kotturi HSR, Li J, Branham-O'Connor M, Yu X, Wagner TE and Wei Y: In vitro and in vivo delivery of novel anticancer fusion protein MULT1E/FasTI via adenoviral vectors. Cancer Gene Ther 17: 164-170, 2010.

14. Law TM, Motzer RJ, Mazumdar M, Sell KW, Walther PJ, O'Connell M, Khan A, Vlamis V, Vogelzang NJ and Bajorin DF: Phase III randomized trial of interleukin-2 with or without lymphokine-activated killer cells in the treatment of patients with advanced renal cell carcinoma. Cancer 76: 824-832, 1995.

15. Meropol NJ, Barresi GM, Fehniger TA, Hitt J, Franklin M and Caligiuri MA: Evaluation of natural killer cell expansion and activation in vivo with daily subcutaneous low-dose interleukin-2 plus periodic intermediate-dose pulsing. Cancer Immunol Immunother 46: 318-326, 1998.

16. Elsässer-Beile U, Gierschner D, Welchner T and Wetterauer U: Different expression of Fas and Fas ligand in tumor infiltrating and peripheral lymphocytes of patients with renal cell carcinomas. Anticancer Res 23 (1A): 433-437, 2003.

17. Bryceson YT, March ME, Ljunggren HG and Long EO: Activation, coactivation, and costimulation of resting human natural killer cells. Immunol Rev 214: 73-91, 2006.

18. Tietje A, Li J, Yu X and Wei Y: MULT1E/mIL-12: A novel bi-functional protein for NK cell activation. Cancer Res 21: 468-475, 2014. 